\title{
THE EFFECT OF ORGANISATION BRAND TO EMPLOYEE ENGAGEMENT THROUGH COMPENSATION AND BENEFIT IN START-UP BUSINESS
}

\author{
Astri Utami Indriyani \\ Ignatius Heruwasto \\ Postgraduate Program in Management Sciences, Universitas Indonesia \\ E-mail: astir.utamiindriyani@yahoo.com
}

\begin{abstract}
The startup business in Indonesia has become a popular way recently. Especially for them who wants to begin their business empire. The business that run by the employee. Employee needs to bring with them the brand of the business because they have to feel the brand as their own identity. Some research find that the more engage the employee, the more productive they become. On the other side, compensation and benefit are also the factor to determine the engagement of the employee. This paper would like to tests the hypothesis that organization brand is having some effect on employee engagement. The evidence collected from 30 employees on various startup businesses. The result shows that organization brand have the effect on employee engagement, as well as the benefit but not with the compensation.
\end{abstract}

Keywords: Compensation, Start-up, Employee engagement 


\section{Astri Utami Indriyani \\ Ignatius Heruwasto}

\section{INTRODUCTION}

Few years now in Indonesia, there are some distractions by some online application that provide any services needed by the citizen. Such as riding, sending packages, food, even home cleaning and beauty services. This kind of disruption on transportation business then known as online ojek. Few players in this business are Gojek from Indonesia, Uber from US or Grab from Malaysia. The business they run named start up business. In an article mentioned by Daily Social Annual Startup Report 2015, appear some interesting facts:

1. $49 \%$ continuity funding given are SEED funding

2. Most start up business are e-commerce

3. East Venture and Lippo Group in Indonesia fund 20 and 7 infestation

4. Subscriber service and financial technology are the most interesting services

5. The biggest problem faced by startup are less funding and talent

Then we are going to see which one is the best startup business in Indonesia. Based on rank given by Startup Ranking, the website who gives ranks to all startup business around the world, number one startup business in Indonesia is Tokopedia, a market place. Followed by Lazada on number two, a retailer. In third place there is Bukalapak, as well as Tokopedia, is a market place. Blibli.com on fourth place, also a retailer. In the fifth place there is Traveloka, a booking website.

An article in Tech in Asia website mention that starts up business in Indonesia is having a huge attention from the government. This proven by an e-commerce roadmap, talking about universal service obligation, incubating programe and tax. The funding for any startup business are also growing. Tokopedia got the investment amount US\$147. The growth of e-commerce also affecting others business chained, that are payment product and shipping method. One thing that must be very interesting for the consumer is that the risen of e-commerce also giving so many discount on different special day. Such as Harbolnas (Hari Belanja Online Nasional or National Online Shop Day).

A lot of startup business in Indonesia introduced them with different identity. Tokopedia closed their campaign with a question like "Sudah Cek Tokopedia belum?" (Have you checked Tokopedia?). The online application mentioned before that is Gojek, open up their application with a very brave statement, "An Ojek for Every Needs". The identity they showed up to customer is an organization strategic choices and expression about. Company identity is the part of corporate brand that is expression and organization identity (Abratt \& Kleyn, 2011). Brand exists to make customer realize the character of the company. 
Specific method needs to be found so the employee will understand in how to internalized the brand into themselves and representing it to the customer (Ahmad et al, 2014).

To make employee able to internalize the brand to them, Boyd \& Sutherland (2006) agree that organization need some characteristics. One of the characteristic is the integration and reward for employee who have commitment to apply brand value to organization (Armstrong, 2000; Mullich, 2003, Boyd \& Sutherland, 2006). Financial rewards are the most considering factor by employees and become a very successful factor (Herman \& Gioia, 2000, Boyd \& Sutherland, 2006).

When employee already has a commitment to the company, engagement arises. Hewitt Associates (2004) mentioned by Anitha J (2013) said that employee engagement is the state in which individuals are emotionally and intellectually committed to the organization or group, as measured by three primary behaviors: say, stay, strive. Employee engagement will increase profit and decrease outcome supported by effectivity, satisfaction, and motivation (Maylett \& Nielsem 2012 in Abel, 2013).

As a consideration of those topics mentioned above, so I decide to write down a research. The question we need to answer are: 1) Is organization brand have effect on employee engagement?; 2) is compensation become a mediating variable between organization brand and employee engagement?; 3) is benefit become a mediating variable between organization brand and employee engagement?; 4) is compensation have effect on employee engagement?; 5) is benefit have effect on employee engagement?; 6) is compensation have effect on organization brand?; 7) is benefit have effect on organization brand?

\section{LITERATURE REVIEW}

Abratt \& Kleyn (2011) said that organization brand is the expressions and images of an organization's identity. They need to have two aspects: corporate expressions and stakeholder images of the organization's identity. While the same term called corporate brand told by Balmer \& Gray (2003) in Abratt \& Kleyn (2011) as ownership sign, tools to build an image, the symbol of key value, to create special identity for company and delightful experience. Organization brand is important asset needed by company to open the chance and prevent the threat (Argenti \& Druckenmiller, 2004). The theory used in this research of organization brand is based on the findings from Punjaisri et al (2009). They said that variables who support organization brand consist of brand identification, brand 


\section{Astri Utami Indriyani \\ Ignatius Heruwasto}

commitment, brand loyalty, and brand performance. Brand identification is an employee's sense of belongin to the brand and a perception of being intertwined with the brand's fate and success (Punjaisri, Wilson, \& Evanschutzky, 2009). Employee who committed will be able to fulfil brand promise because they have emotional proximity with the brand (Thomson et al, 1999; Punjaisri \& Wilson, 2010). Brand loyalty is defined as an employee's willingness to remain with the present brand (PUnjaisri, Wilson, \& Evanschutzky, 2009). And the last, brand performance is the extent to which an employee performs his/her role in the brand promise delivery based on the brand standards in which the brand values are embedded (Punjaisri et al, 2009).

Martocchio (2015) said that compensation is intrinsic and extrinsic reward accepted by employee after they fulfilled their job. Intrinsic compensation show the mindset of an employee while extrinsic compensation is about monetary and non-monetary reward. Milkovich et al (2014) agree that compensation is the part of feedback got by the employee based on everything they have done on work. The type of compensation included in this research is base pay, cost-of-living adjustments, seniority pay, incentives, long-term incentives, and person-focused pay. Base pays actually some money which always gets by employee as long as they are working. It usually depends on stages of skill and the responsibility needed on some job (Martocchio, 2015). Cost - of - living adjustment is the adjustment cost given by company to the living cost of the employee. The adjustments usually count based on consumer price index (CPI). Seniority pay is the additional pay given by company as a reward to duration of working of some employee. Incentives and longterm incentives is the same concept but different in time span. Incentives given if employee successfully done some special task. Incentives is some kind of variable pay. While ordinary incentives given once in some period (monthly), long-term incentives are annually. Company gives this long-term incentive to them who give outstanding contribution to the company. The last is person-focused pay. These rewards are for them who have specialty on skill. Whether their skill will be used in their job or not, as long as they have a deep or broad skill of something.

Benefit is another term used in this research. Benefit defined by Murphy (2010) as rewards got by employee in a different kind than compensation. Usually in concept of income protection, insurance, paid time off, and any other. First, income protection, is the protection given by country to someone who become disable or unable to work. The fund collected by employer who gives the fund to some institution (Milkovich, 2014). Second, work-life balance. To provide the equilibrium between work life and private life, employer provide certain facilities. Company also give different allowances such as bill allowances, 


\section{Jurnal Manajemen Teori dan Terapan \\ Tahun 10. No. 2, Agustus 2017}

transportation allowance, and lunch allowance. Fourth, paid time off. A chance employee gets paid even though they are not working (Martocchio, 2015). Some type of paid time off are annual, menstruation, married, pregnant, and any other. The last is services. Different services provide by the company. It is including reimbursement of school fee or parents care.

Kahn (1990, in Condos, 2016) defined employee engagement as harnessing of organization members' selves to their work roles, in engagement, people employ and express themselves physically, cognitively, and emotionally during role performances. Buckingham and Coffman (1999) having an opinion of engagement with the right people in the right roles with the right managers drive employee engagement. Research by Corporate Leadership Council explain engagement as positive emotional connection to an employee's work, affective, normative, and continuance commitment, and inspired to go above and beyond the call of duty to help meet business goals. AON Hewitt is an institution who frequently releasing a research result of employee engagement. AON Hewitt (2015) divides employee engagement into three dimensions that is Say, Stay, and Strive. Say is about how employee talking about the company they work in. Stay is about anything can help employee to continuously working in this company. While Strive encourages employee to release their best effort and work as hard as they can. Corporate Leadership Council (2004) explained employee engagement as emotional positive relationship between employee and the job, affective commitment, normative, and continuity. There are twi dimensions on employee engagement by Corporate Leadership Council: 1) Rational Commitment, the extent to which employees believe that managers, teams, or organizations are in their selfinterest (financial, developmental, or professional); 2) Emotional Commitment, the extent to which employees values, enjoy, and believe in their jobs, managers, teams, or organizations.

Paul Graham on startupbisnis.com talks about startup. He said that "startups usually involve technology, so much so that the phrase 'high-tech startup' is almost redundant. A startup is a small company that takes on a hard technical problem. Economically, you can think of a startup as a way to compress your whole working-life into a few years. Instead of working at a low intensity for forty years, you work as hard as you possibly can for four this pays especially well in technology, where you earn a premium for working fast. Startups offer anyone a way to be in a situation with measurement and leverage they allow measurement because they are small and they offer leverage because they make money by inventing new technology." 


\section{Astri Utami Indriyani}

Ignatius Heruwasto

\section{THE METHODOLOGY AND MODEL}

To test the hypotheses I have conducted an empirical study and collecting 30 employees from different set of start-up business in Indonesia. The model in this research is as mention below and we proposed three group of hypotheses consist of one dependent variable, one independent variable, and two mediating variable.

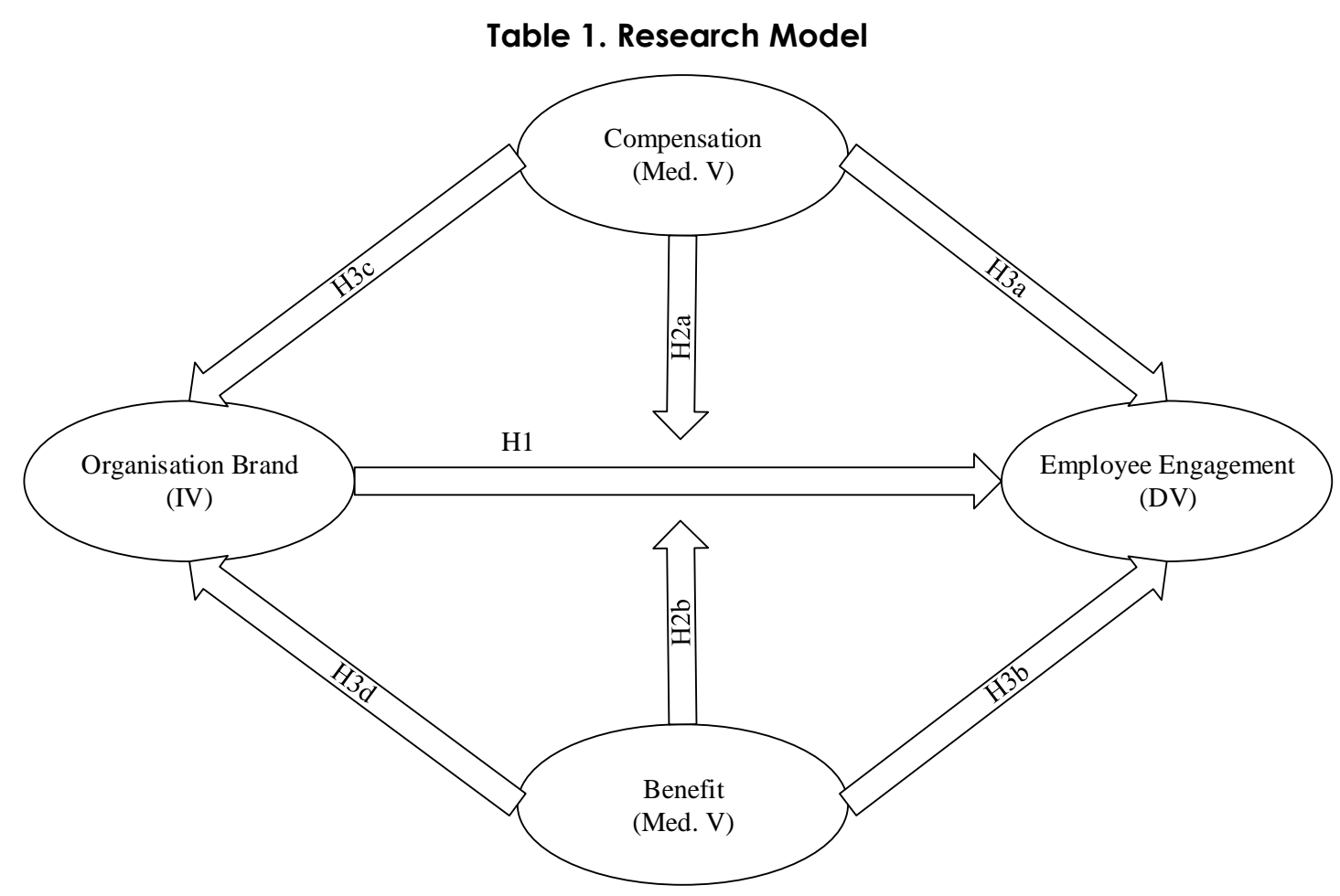

The research done in Pakistan in 2014 about brand of a service company by Ahmad and friends. This research resulting in conclusions those brands have positive correlation with employee engagement (Ahmad et al, 2014). One of the ways to develop internal branding in a company is through five key factors (Blumenthal, 2011). There are clarity, commitment, communications, culture, and compensation. While on the concept of employee engagement, there are some determinant factors about the emergence of the variable. The determinants are leadership, team and co-worker, training and career development, compensation, organizational policies, and workplace well-being. From those explanations, we agree that in both organization brand and employee engagement, they consist of same factor that is compensation. Talking about compensation, it will always goes alongside benefit. Because those two things are the remuneration point given to the employee but in different kind of things. 


\section{Jurnal Manajemen Teori dan Terapan \\ Tahun 10. No. 2, Agustus 2017}

Ahmad (2014) said that when an employee lives the brand, they able to get the instruction clearly about the task and believe in such effort from company to satisfied customer and employee. This will result in employee commitment to the company (Punjaisri, Wilson, Evanschitzky, 2009; Mitchell, 2002). So the first hypothesis proposed in this research is:

H1: Organization brand have effect on employee engagement.

Anitha J (2013) in her research told us about compensation as one of the factors facilitating in employee engagement. Together with six other factors, work environment, leadership, teamd and co-worker, training and career development, organizational policies, and workplace well-being. Abel (2013) is also agree that employee engagement will increase profit and reduces cost by satisfaction in employee (Maylett \& Nielsen 2012). Boyd \& Sutherland (2006) did the research about how employee internalized the brand of company they work at. They found that one of the factors correlating with employee living the brand is the appearance of compensation. On research did by Blumenthal on 2011 ebout employee quality of life, to internalized brand to every self of the employee is through five factors, Those five factors are clarity, commitment, communication, culture, and compensation, The we have our second set of hypothesis:

H2a: Compensation is a mediating variable in the effect of organization brand to employee engagement

H2b: Benefit is a mediating variable in the effect of organization brand to employee engagement

As we remember that compensation and benefit actually able to determine either organization brand and employee engagement separately. As define above by Anitha J (2013) about compensation as one determinant factor in employee engagement and Blumenthal (2011) also said that compensation and benefit is one of the factors affecting organization brand, so we came to the conclusions of the third set of hypotheses:

H3a : Compensation is the factor affecting employee engagement

H3b : Benefit is the factor affecting employee engagement

$\mathrm{H} 3 \mathrm{C}$ : Compensation is the factor affecting organization brand

H3d : Benefit is the factor affecting organization brand

All these hypotheses tested in this research to start-up business emerging in Indonesia. To answer the question of the research, we use the quantitative research method. Primary data collected from first hand respondent. The data that has been collected treated using SPSS 16.0 version. Questionnaire chosen by consideration as most easy to get research tools and respondents will easily answer as well. The research goes to everyone who work in start- 


\section{Astri Utami Indriyani}

\section{Ignatius Heruwasto}

up business regardless their gender and duration of work. Through non-random sampling method which whoever can be a participant as long as they are work in start-up business. At the preparation stage, researcher needs to prepare the concept, the questionnaire, and the data collecting way. Questionnaire in this research originally from other research. Organisation brand questionnaire is an adaptation of Punjaisri et al (2009) with some translation to fit into Indonesian language and understanding. While the questionnaire of employee engagement is the development of Kahn (1990). Scale in the questionnaire is Likert scale with 6 rates from 1 as Very Inappropriate to 6 as Very Appropriate. Researcher purposely erase middle rate to avoid central tendency. About the compensation and benefit, by the theory of Martocchio, researcher has developed own question about type of compensation and benefit employee can get in their company. To give richness in the research, we also provide descriptive question about gender, age, duration of work, level of job, and type of work.

Organisation brand questionnaire in this research consist four dimension including brand identification, brand commitment, brand loyalty, and brand performance. Employee engagement consist three dimension including physical engagement, cognitive engagement, and emotional engagement. Compensation questions are all about external compensation because it is physically form. Last, benefit questions including income protection, work life balance, allowances, paid time off, and services.

To ensure that this questionnaire is valid and reliable to be used in the research, first of all we do pretest research. Validity and reliability coefficient result in this questionnaire are overall valid and reliable but in some items need to be revised. The results are below.

Table 2. Validity and Reliability Test Result

\begin{tabular}{|l|l|c|c|c|l|l|}
\hline Concept & Item & KMO & $\begin{array}{c}\text { Factor } \\
\text { Loading }\end{array}$ & $\begin{array}{c}\text { Alpha- } \\
\text { Cronbach }\end{array}$ & Conclusion & Revision \\
\hline Brand & BRID1 & 0.843 & 0.898 & 0.943 & Valid. & Fix \\
& BRID2 & & 0.883 & & Reliable & Fix \\
& BRID3 & & 0.903 & & Valid. & Fix \\
& BRID4 & & 0.850 & & Reliable & Fix \\
& BRID5 & & 0.936 & & Valid. & Fix \\
& BRID6 & & 0.921 & & Reliable & Fix \\
& BRID7 & & 0.433 & & Valid. & Change \\
& BRID8 & & 0.889 & & Reliable & words \\
\hline
\end{tabular}




\begin{tabular}{|c|c|c|c|c|c|c|}
\hline & & & & & $\begin{array}{l}\text { Valid. } \\
\text { Reliable } \\
\text { Valid. } \\
\text { Reliable } \\
\text { Invalid, } \\
\text { Reliable } \\
\text { Valid. } \\
\text { Reliable }\end{array}$ & Fix \\
\hline $\begin{array}{l}\text { Brand } \\
\text { Commitment }\end{array}$ & $\begin{array}{l}\text { BRCO1 } \\
\text { BRCO2 } \\
\text { BRCO3 } \\
\text { BRCO4 }\end{array}$ & 0.537 & $\begin{array}{l}0.873 \\
0.856 \\
0.622 \\
0.599\end{array}$ & 0.721 & $\begin{array}{l}\text { Valid. } \\
\text { Reliable } \\
\text { Valid. } \\
\text { Reliable } \\
\text { Valid. } \\
\text { Reliable } \\
\text { Valid. } \\
\text { Reliable }\end{array}$ & $\begin{array}{l}\text { Fix } \\
\text { Fix } \\
\text { Fix } \\
\text { Fix }\end{array}$ \\
\hline Brand Loyalty & $\begin{array}{l}\text { BRLO1 } \\
\text { BRLO2 } \\
\text { BRLO3 }\end{array}$ & 0.555 & $\begin{array}{l}0.912 \\
0.641 \\
0.844\end{array}$ & 0.722 & $\begin{array}{l}\text { Valid. } \\
\text { Reliable } \\
\text { Valid. } \\
\text { Reliable } \\
\text { Valid. } \\
\text { Reliable }\end{array}$ & $\begin{array}{l}\text { Fix } \\
\text { Fix } \\
\text { Fix }\end{array}$ \\
\hline $\begin{array}{l}\text { Brand } \\
\text { Performance }\end{array}$ & $\begin{array}{l}\text { BRPElz } \\
\text { BRPE2 } \\
\text { BRPE3 } \\
\text { BRPE4 } \\
\text { BRPE5 }\end{array}$ & 0.572 & $\begin{array}{l}0.737 \\
0.272 \\
0.802 \\
0.687 \\
0.803\end{array}$ & 0.667 & $\begin{array}{l}\text { Valid. } \\
\text { Reliable } \\
\text { Invalid. } \\
\text { Reliable } \\
\text { Valid. } \\
\text { Reliable } \\
\text { Valid. } \\
\text { Reliable } \\
\text { Valid. } \\
\text { Reliable }\end{array}$ & $\begin{array}{l}\text { Fix } \\
\text { Change } \\
\text { words } \\
\text { Fix } \\
\text { Fix } \\
\text { Fix }\end{array}$ \\
\hline $\begin{array}{l}\text { Physical } \\
\text { Engagement }\end{array}$ & $\begin{array}{l}\text { PHEN1 } \\
\text { PHEN2 } \\
\text { PHEN3 } \\
\text { PHEN4 } \\
\text { PHEN5 }\end{array}$ & 0.741 & $\begin{array}{l}0.846 \\
0.921 \\
0.869 \\
0.903 \\
0.690\end{array}$ & 0.916 & $\begin{array}{l}\text { Valid. } \\
\text { Reliable } \\
\text { Valid. } \\
\text { Reliable } \\
\text { Valid. }\end{array}$ & $\begin{array}{l}\text { Fix } \\
\text { Fix } \\
\text { Fix } \\
\text { Fix } \\
\text { Fix }\end{array}$ \\
\hline
\end{tabular}




\begin{tabular}{|c|c|c|c|c|c|c|}
\hline & PHEN6 & & 0.804 & & $\begin{array}{l}\text { Reliable } \\
\text { Valid. } \\
\text { Reliable } \\
\text { Valid. } \\
\text { Reliable } \\
\text { Valid. } \\
\text { Reliable }\end{array}$ & Fix \\
\hline $\begin{array}{l}\text { Cognitive } \\
\text { Engagement }\end{array}$ & $\begin{array}{l}\text { COEN1 } \\
\text { COEN2 } \\
\text { COEN3 } \\
\text { COEN4 } \\
\text { COEN5 } \\
\text { COEN6 }\end{array}$ & 0.889 & $\begin{array}{l}0.908 \\
0.871 \\
0.935 \\
0.869 \\
0.818 \\
0.865\end{array}$ & 0.939 & $\begin{array}{l}\text { Valid. } \\
\text { Reliable } \\
\text { Valid. } \\
\text { Reliable } \\
\text { Valid. } \\
\text { Reliable } \\
\text { Valid. } \\
\text { Reliable } \\
\text { Valid. } \\
\text { Reliable } \\
\text { Valid. } \\
\text { Reliable }\end{array}$ & $\begin{array}{l}\text { Fix } \\
\text { Fix } \\
\text { Fix } \\
\text { Fix } \\
\text { Fix } \\
\text { Fix }\end{array}$ \\
\hline $\begin{array}{l}\text { Emotional } \\
\text { Engagement }\end{array}$ & $\begin{array}{l}\text { EMEN1 } \\
\text { EMEN2 } \\
\text { EMEN3 } \\
\text { EMEN4 } \\
\text { EMEN5 } \\
\text { EMEN6 }\end{array}$ & 0.734 & $\begin{array}{l}0.829 \\
0.781 \\
0.936 \\
0.832 \\
0.821 \\
0.723\end{array}$ & 0.899 & $\begin{array}{l}\text { Valid. } \\
\text { Reliable } \\
\text { Valid. } \\
\text { Reliable } \\
\text { Valid. } \\
\text { Reliable } \\
\text { Valid. } \\
\text { Reliable } \\
\text { Valid. } \\
\text { Reliable } \\
\text { Valid. } \\
\text { Reliable }\end{array}$ & $\begin{array}{l}\text { Fix } \\
\text { Fix } \\
\text { Fix } \\
\text { Fix } \\
\text { Fix } \\
\text { Fix }\end{array}$ \\
\hline $\begin{array}{l}\text { Extrinsic } \\
\text { Compensation }\end{array}$ & $\begin{array}{l}\text { EXCO1 } \\
\text { EXCO2 } \\
\text { EXCO3 } \\
\text { EXCO4 } \\
\text { EXCO5 }\end{array}$ & 0.611 & $\begin{array}{l}0.560 \\
0.608 \\
0.663 \\
0.669 \\
0.499\end{array}$ & 0.802 & $\begin{array}{l}\text { Valid. } \\
\text { Reliable } \\
\text { Valid. } \\
\text { Reliable } \\
\text { Valid. }\end{array}$ & $\begin{array}{l}\text { Fix } \\
\text { Fix } \\
\text { Fix } \\
\text { Fix } \\
\text { Fix }\end{array}$ \\
\hline
\end{tabular}


Jurnal Manajemen Teori dan Terapan

Tahun 10. No. 2, Agustus 2017

\begin{tabular}{|c|c|c|c|c|c|c|}
\hline & $\begin{array}{l}\text { EXCO6 } \\
\text { EXCO7 } \\
\text { EXCO } 8 \\
\text { EXCO9 } \\
\text { EXCO1 } \\
0 \\
\text { EXCO } 1 \\
1\end{array}$ & & $\begin{array}{l}0.748 \\
0.830 \\
0.272 \\
0.390 \\
0.421 \\
0.778\end{array}$ & & $\begin{array}{l}\text { Reliable } \\
\text { Valid. } \\
\text { Reliable } \\
\text { Valid. } \\
\text { Reliable } \\
\text { Valid. } \\
\text { Reliable } \\
\text { Valid. } \\
\text { Reliable } \\
\text { Invalid. } \\
\text { Reliable } \\
\text { Invalid. } \\
\text { Reliable } \\
\text { Invalid. } \\
\text { Reliable } \\
\text { Invalid. } \\
\text { Reliable }\end{array}$ & $\begin{array}{l}\text { Fix } \\
\text { Fix } \\
\text { Change } \\
\text { words } \\
\text { Change } \\
\text { words } \\
\text { Change } \\
\text { words } \\
\text { Delete }\end{array}$ \\
\hline $\begin{array}{l}\text { Income } \\
\text { protection }\end{array}$ & BENE2 & & & & Unidentified & $\begin{array}{l}\text { Add } \\
\text { another } 4 \\
\text { item }\end{array}$ \\
\hline $\begin{array}{l}\text { Work-life } \\
\text { balance }\end{array}$ & $\begin{array}{l}\text { BENE20 } \\
\text { BENE21 } \\
\text { BENE22 } \\
\text { BENE24 } \\
\text { BENE25 }\end{array}$ & 0.465 & $\begin{array}{c}0.508 \\
0.840 \\
0.388 \\
-0.593 \\
0.634\end{array}$ & 0.224 & $\begin{array}{l}\text { Valid. } \\
\text { Unreliable } \\
\text { Valid. } \\
\text { Unreliable } \\
\text { Valid. } \\
\text { Unreliable } \\
\text { Valid. } \\
\text { Unreliable } \\
\text { Valid. } \\
\text { Unreliable }\end{array}$ & $\begin{array}{l}\text { Change } \\
\text { words } \\
\text { Change } \\
\text { words } \\
\text { Change } \\
\text { words } \\
\text { Change } \\
\text { words } \\
\text { Change } \\
\text { words }\end{array}$ \\
\hline Allowances & $\begin{array}{l}\text { BENE1 } \\
\text { BENE10 } \\
\text { BENE11 } \\
\text { BENE12 } \\
\text { BENE13 } \\
\text { BENE } 14 \\
\text { BENE15 }\end{array}$ & 0.510 & $\begin{array}{l}-0.395 \\
0.239 \\
0.712 \\
0.504 \\
0.819 \\
0.765 \\
0.692\end{array}$ & 0.757 & $\begin{array}{l}\text { Invalid. } \\
\text { Reliable } \\
\text { Invalid. } \\
\text { Reliable } \\
\text { Valid. } \\
\text { Reliable } \\
\text { Valid. }\end{array}$ & $\begin{array}{l}\text { Change } \\
\text { words } \\
\text { Delete } \\
\text { Fix } \\
\text { Fix } \\
\text { Fix } \\
\text { Fix }\end{array}$ \\
\hline
\end{tabular}




\begin{tabular}{|c|c|c|c|c|c|c|}
\hline & $\begin{array}{l}\text { BENE16 } \\
\text { BENE17 } \\
\text { BENE18 }\end{array}$ & & $\begin{array}{l}0.625 \\
0.801 \\
0.695\end{array}$ & & $\begin{array}{l}\text { Reliable } \\
\text { Valid. } \\
\text { Reliable } \\
\text { Valid. } \\
\text { Reliable } \\
\text { Valid. } \\
\text { Reliable } \\
\text { Valid. } \\
\text { Reliable } \\
\text { Valid. } \\
\text { Reliable } \\
\text { Valid. } \\
\text { Reliable }\end{array}$ & $\begin{array}{l}\text { Fix } \\
\text { Fix } \\
\text { Fix } \\
\text { Fix }\end{array}$ \\
\hline Paid time off & $\begin{array}{l}\text { BENE3 } \\
\text { BENE4 } \\
\text { BENE5 } \\
\text { BENE6 } \\
\text { BENE7 } \\
\text { BENE8 } \\
\text { BENE9 }\end{array}$ & 0.747 & $\begin{array}{l}0.889 \\
0.939 \\
0.765 \\
0.902 \\
0.129 \\
-0.079 \\
0.804\end{array}$ & 0.673 & $\begin{array}{l}\text { Valid. } \\
\text { Reliable } \\
\text { Valid. } \\
\text { Reliable } \\
\text { Valid. } \\
\text { Reliable } \\
\text { Valid. } \\
\text { Reliable } \\
\text { Invalid. } \\
\text { Reliable } \\
\text { Invalid. } \\
\text { Reliable } \\
\text { Valid. } \\
\text { Reliable }\end{array}$ & $\begin{array}{l}\text { Fix } \\
\text { Fix } \\
\text { Fix } \\
\text { Fix } \\
\text { Change } \\
\text { words } \\
\text { Change } \\
\text { words } \\
\text { Fix }\end{array}$ \\
\hline Services & $\begin{array}{l}\text { BENE19 } \\
\text { BENE23 } \\
\text { BENE26 } \\
\text { BENE27 }\end{array}$ & 0.736 & $\begin{array}{l}0.538 \\
0.972 \\
0.976 \\
0.932\end{array}$ & 0.842 & $\begin{array}{l}\text { Valid. } \\
\text { Reliable } \\
\text { Valid. } \\
\text { Reliable } \\
\text { Valid. } \\
\text { Reliable } \\
\text { Valid. } \\
\text { Reliable }\end{array}$ & $\begin{array}{l}\text { Fix } \\
\text { Fix } \\
\text { Fix } \\
\text { Fix }\end{array}$ \\
\hline
\end{tabular}


Some items which need to be revised resulting in a brand new questionnaire. Here are the questions used in the real data collection.

Table 3. Item in Questionnaire

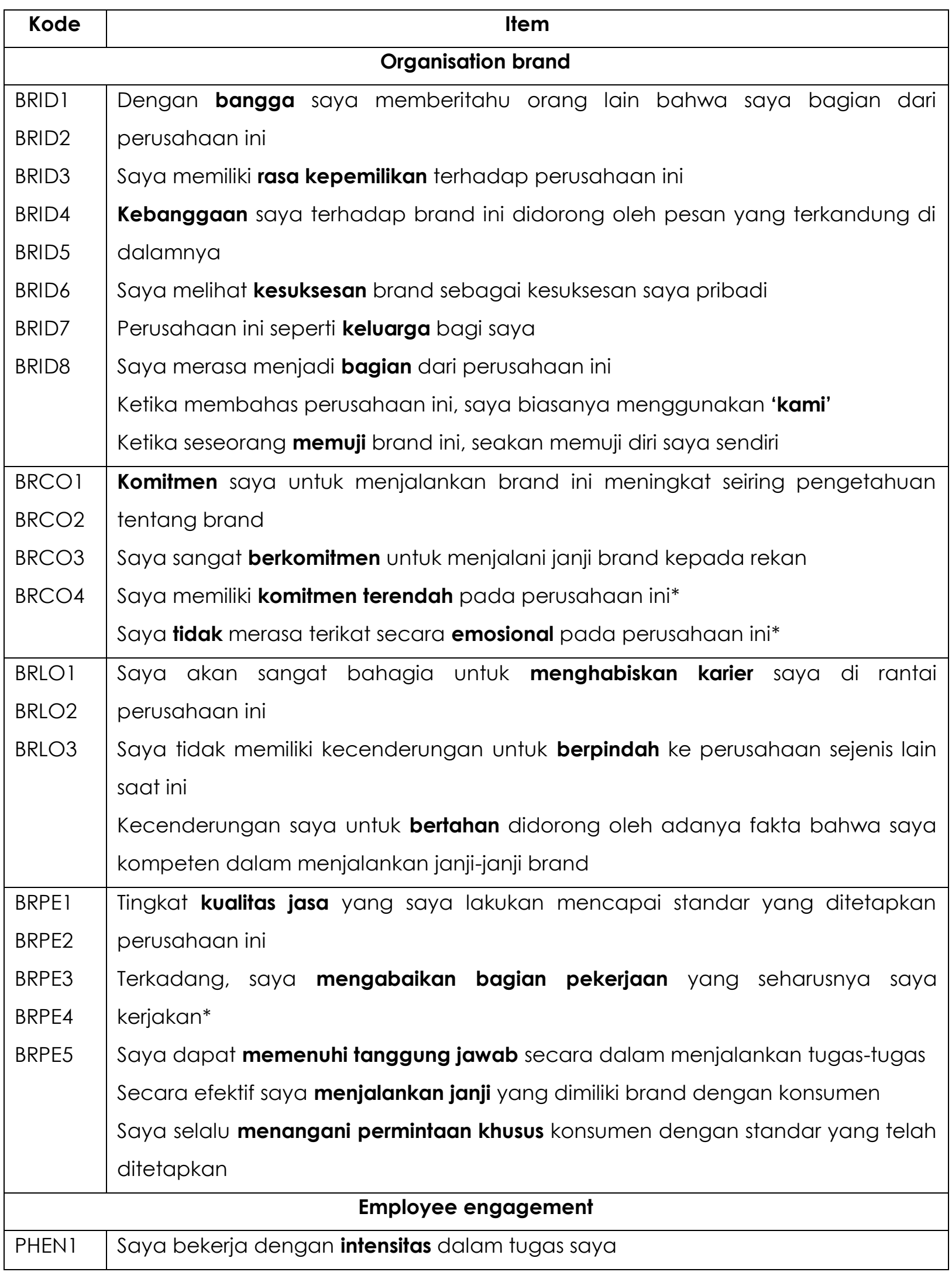




\section{Astri Utami Indriyani}

Ignatius Heruwasto

\begin{tabular}{|c|c|}
\hline PHEN2 & Saya mengerahkan usaha semaksimal mungkin pada tugas saya \\
\hline PHEN3 & Saya mencurahkan banyak energi pada tugas saya \\
\hline PHEN4 & Saya berusaha keras untuk melakukan yang terbaik dalam tugas saya \\
\hline PHEN5 & Saya berjuang sekeras yang saya bisa untuk menyelesaikan tugas saya \\
\hline PHEN6 & Saya mengerahkan energi yang banyak dalam tugas \\
\hline COEN1 & Saya antusias mengenai tugas saya \\
\hline COEN2 & Saya merasa penuh semangat tentang tugas saya \\
\hline COEN3 & Saya tertarik pada tugas saya \\
\hline COEN4 & Saya bangga terhadap tugas saya \\
\hline COEN5 & Saya merasa positif tentang tugas saya \\
\hline COEN6 & Saya merasa bersemangat tentang tugas saya \\
\hline EMEN1 & Pikiran saya fokus dalam tugas saya \\
\hline EMEN2 & Saya memberikan perhatian penuh pada tugas saya \\
\hline EMEN3 & Saya berkonsentrasi dalam tugas saya \\
\hline EMEN4 & Saya memberikan fokus yang besar dalam tugas saya \\
\hline EMEN5 & Saya terserap oleh tugas saya \\
\hline EMEN6 & Saya mencurahkan banyak perhatian pada tugas saya \\
\hline \multicolumn{2}{|r|}{ Compensation } \\
\hline EXCO1 & Gaji pokok yang saya dapatkan sudah sesuai \\
\hline EXCO2 & Saya mendapatkan insentif setiap bulan atas kinerja saya \\
\hline EXCO3 & Saya mendapatkan bonus tahunan \\
\hline $\mathrm{EXCO} 4$ & Saya mendapatkan pendapatan tambahan terkait lama bekerja \\
\hline EXCO5 & Saya mendapatkan pendapatan untuk tambahan biaya hidup \\
\hline EXCO6 & Gaji yang saya dapatkan setiap bulan mencakup tunjangan makan \\
\hline EXCO7 & Gaji yang saya dapatkan setiap bulan mencakup tunjangan pulsa \\
\hline EXCO8 & Gaji yang saya dapatkan setiap bulan mencakup tunjangan penggantian \\
\hline EXCO9 & transportasi \\
\hline EXCO 1 & Gaji yang saya dapatkan setiap bulan mencakup tunjangan jabatan \\
\hline 0 & Gaji yang saya dapatkan setiap bulan mencakup asuransi kesehatan \\
\hline \multicolumn{2}{|r|}{ Benefit } \\
\hline INPRI & Gaji saya disisihkan untuk tabungan di hari tua \\
\hline INPR2 & Perusahaan saya memberikan perlindungan pada karyawan yang disable \\
\hline INPR3 & $\begin{array}{l}\text { Perusahaan saya memberikan perlindungan pada karyawan yang tidak dapat } \\
\text { bekerja lagi }\end{array}$ \\
\hline WOBAl & Fasilitas yang diberikan perusahaan termasuk produk perusahaan secara gratis \\
\hline WOBA2 & Fasilitas yang diberikan perusahaan termasuk makan siang gratis \\
\hline
\end{tabular}




\begin{tabular}{|c|c|}
\hline $\begin{array}{l}\text { WOBA3 } \\
\text { WOBA4 } \\
\text { WOBA5 }\end{array}$ & $\begin{array}{l}\text { Fasilitas yang diberikan perusahaan termasuk tempat olahraga/g } \\
\text { Fasilitas yang diberikan perusahaan termasuk minuman gratis } \\
\text { Fasilitas yang diberikan perusahaan termasuk medical check up }\end{array}$ \\
\hline $\begin{array}{l}\text { ALLO1 } \\
\text { ALLO2 } \\
\text { ALLO3 } \\
\text { ALLO4 } \\
\text { ALLO5 } \\
\text { ALLO6 } \\
\text { ALLO7 } \\
\text { ALLO8 } \\
\text { ALLO9 }\end{array}$ & $\begin{array}{l}\text { Perusahaan tempat saya bekerja memberikan tunjangan penempatan kepada } \\
\text { karyawannya } \\
\text { Perusahaan tempat saya bekerja memberikan tunjangan bensin kepada } \\
\text { karyawannya } \\
\text { Perusahaan tempat saya bekerja memberikan tunjangan pulsa kepada } \\
\text { karyawannya } \\
\text { Perusahaan tempat saya bekerja memberikan tunjangan perabotan kepada } \\
\text { karyawannya } \\
\text { Perusahaan tempat saya bekerja memberikan tunjangan tugas khusus kepada } \\
\text { karyawannya } \\
\text { Perusahaan tempat saya bekerja memberikan tunjangan makan kepada } \\
\text { karyawannya } \\
\text { Perusahaan tempat saya bekerja memberikan tunjangan kendaraan kepada } \\
\text { karyawannya } \\
\text { Perusahaan tempat saya bekerja memberikan tunjangan keamanan kepada } \\
\text { karyawannya }\end{array}$ \\
\hline $\begin{array}{l}\text { PATO } 1 \\
\text { PATO2 } \\
\text { PATO3 } \\
\text { PATO4 } \\
\text { PATO5 } \\
\text { PATO6 } \\
\text { PATO7 }\end{array}$ & $\begin{array}{l}\text { Cuti yang diberikan oleh perusahaan mencakup cuti tahunan } \\
\text { Cuti yang diberikan oleh perusahaan mencakup cuti ibadah } \\
\text { Cuti yang diberikan oleh perusahaan mencakup cuti menikah } \\
\text { Cuti yang diberikan oleh perusahaan mencakup cuti panjang setelah sekian } \\
\text { tahun bekerja } \\
\text { Cuti yang diberikan oleh perusahaan mencakup cuti haid bagi perempuan } \\
\text { Saat saya cuti, saya tetap dibayar }\end{array}$ \\
\hline $\begin{array}{l}\text { SERV1 } \\
\text { SERV2 } \\
\text { SERV3 } \\
\text { SERV4 }\end{array}$ & $\begin{array}{l}\text { Fasilitas yang diberikan perusahaan termasuk biaya sekolah anak } \\
\text { Fasilitas yang diberikan perusahaan termasuk perawatan orang tua } \\
\text { Fasilitas yang diberikan perusahaan termasuk cicilan kendaraan }\end{array}$ \\
\hline
\end{tabular}

As the data has been collected using questions above, then we search for the answer of research question using regression method. This method was chosen because we want to know the effect of organization brand to employee engagement and how is the role of compensation and benefit on that relationship. On the other side, we also need to answer 


\section{Astri Utami Indriyani}

Ignatius Heruwasto

the question whether compensation and benefit are affecting organisation and brand as independently as well.

\section{THE FINDINGS}

In the calculation of the effect of Organization Brand to Employee Engagement using linier regression method, determinant coefficient is 0.258 which means dependent variable employee engagement is $25,8 \%$ defined by independent variable organization brand while the rest are another variable we don't say in this research. The significance amount s $0.004<$ 0.05 so the regression model can be accepted to predict employee engagement variable.

Table 4. SPSS Result of Linier Regression Organization Brand to Employee Engagement

Model Summary

\begin{tabular}{|l|r|r|r|r|}
\hline Model & \multicolumn{1}{|c|}{$R$} & R Square & \multicolumn{1}{c|}{$\begin{array}{c}\text { Adjusted R } \\
\text { Square }\end{array}$} & $\begin{array}{r}\text { Std. Error of } \\
\text { the Estimate }\end{array}$ \\
\hline 1 & $.508 \mathrm{a}$ & .258 & .232 & 9.974 \\
\hline
\end{tabular}

a. Predictors: (Constant), BRANX

ANOVAb

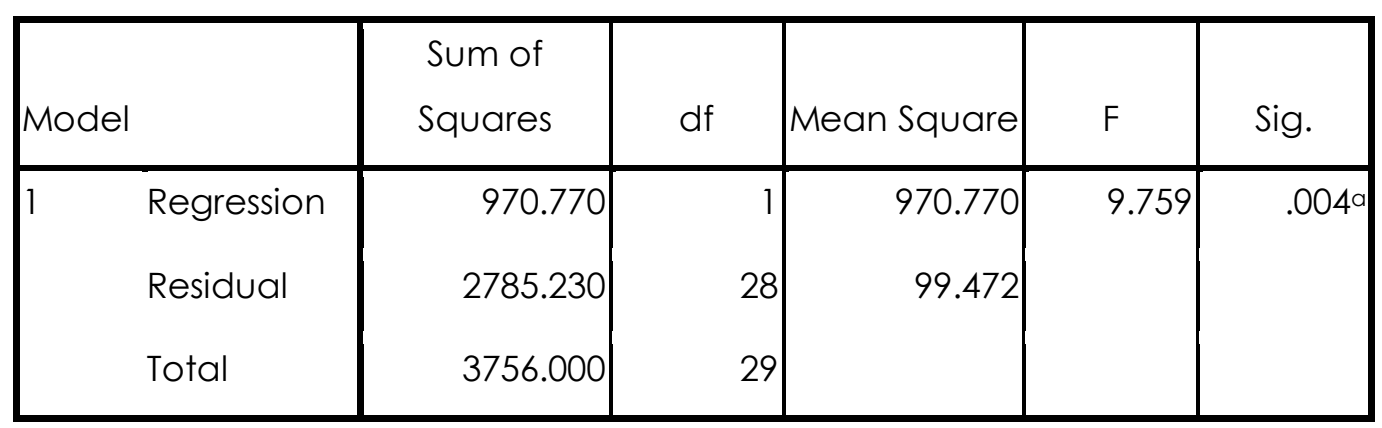

a. Predictors: (Constant), BRANX

b. Dependent Variable: ENGAY 


\section{Coefficientsa}

\begin{tabular}{|c|c|c|c|c|c|}
\hline \multirow[b]{2}{*}{ Model } & \multicolumn{2}{|c|}{$\begin{array}{c}\text { Unstandardized } \\
\text { Coefficients }\end{array}$} & \multirow{2}{*}{\begin{tabular}{|c|}
$\begin{array}{c}\text { Standardized } \\
\text { Coefficients }\end{array}$ \\
Beta
\end{tabular}} & \multirow[b]{2}{*}{$t$} & \multirow[b]{2}{*}{ Sig. } \\
\hline & B & Std. Error & & & \\
\hline (Constant) & 34.269 & 15.705 & & 2.182 & .038 \\
\hline BRANX & .549 & .176 & .508 & 3.124 & .004 \\
\hline
\end{tabular}

a. Dependent Variable: ENGAY

While using double regression method to see the relationship of those variables, we got the result that $r$ of correlation between organisation brand is 0.508 with significance coefficient in $0.002<0.05$. Compensation variable have $r$ amount in 0.087 with significance coefficient $0.323>0.05$. And Benefit variable got $r$ amount in 0.427 with significance coefficient in 0.009 $<0.05$.

Determinant coefficient of organisation brand and engagement is 0.258 which means the effect of organisation brand are $25,8 \%$. In model 2, determinant coefficient between benefit and compensation is $13,4 \%(39,2 \%$ - $25,8 \%)$. The last, determinant coefficient of compensation and employee engagement is $25,8 \%$.

Table 5. SPSS Result of Multiply Regression Organization Brand, Compensation, Benefit, to Employee Engagement

Coefficientsa

\begin{tabular}{|c|c|c|c|c|c|}
\hline \multirow[b]{2}{*}{ Model } & \multicolumn{2}{|c|}{$\begin{array}{l}\text { Unstandardized } \\
\text { Coefficients }\end{array}$} & \multirow{2}{*}{\begin{tabular}{|c|}
$\begin{array}{c}\text { Standardized } \\
\text { Coefficients }\end{array}$ \\
Beta
\end{tabular}} & \multirow[b]{2}{*}{$\dagger$} & \multirow[b]{2}{*}{ Sig. } \\
\hline & B & Std. Error & & & \\
\hline (Constant) & 34.269 & 15.705 & & 2.182 & .038 \\
\hline BRANX & .549 & .176 & .508 & 3.124 & .004 \\
\hline
\end{tabular}

a. Dependent Variable: ENGAY 


\section{Correlations}

\begin{tabular}{|ll|r|r|r|r|}
\hline & & ENGAY & BRANX & COMPX1 & BENEX2 \\
\hline Pearson & ENGAY & 1.000 & .508 & .087 & .427 \\
& BRANX & .508 & 1.000 & .443 & .127 \\
& COMPX1 & .087 & .443 & 1.000 & .629 \\
& BENEX2 & .427 & .127 & .629 & 1.000 \\
\hline Sig. (1-tailed) & ENGAY &. & .002 & .323 & .009 \\
& BRANX & .002 &. & .007 & .252 \\
& COMPX1 & .323 & .007 &. & .000 \\
& BENEX2 & .009 & .252 & .000 & \\
& ENGAY & 30 & 30 & 30 & 30 \\
& BRANX & 30 & 30 & 30 & 30 \\
& COMPX1 & 30 & 30 & 30 & 30 \\
& BENEX2 & 30 & 30 & 30 & 30 \\
\hline
\end{tabular}

Model Summary

\begin{tabular}{|l|r|r|r|r|}
\hline Model & $R$ & R Square & \multicolumn{1}{c|}{$\begin{array}{c}\text { Adjusted R } \\
\text { Square }\end{array}$} & $\begin{array}{c}\text { Std. Error of } \\
\text { the Estimate }\end{array}$ \\
\hline 1 & $.508 \mathrm{a}$ & .258 & .232 & 9.974 \\
2 & $.626 \mathrm{~b}$ & .392 & .347 & 9.199 \\
3 & $.806 \mathrm{c}$ & .650 & .609 & 7.112 \\
\hline
\end{tabular}

a. Predictors: (Constant), BRANX

b. Predictors: (Constant), BRANX, BENEX2

c. Predictors: (Constant), BRANX, BENEX2, COMPX1 
ANOVAd

\begin{tabular}{|c|c|c|c|c|c|c|}
\hline \multicolumn{2}{|c|}{ Model } & $\begin{array}{l}\text { Sum of } \\
\text { Squares }\end{array}$ & $d f$ & Mean Square & $\mathrm{F}$ & Sig. \\
\hline 1 & $\begin{array}{l}\text { Regression } \\
\text { Residual } \\
\text { Total }\end{array}$ & $\begin{array}{r}970.770 \\
2785.230 \\
3756.000\end{array}$ & $\begin{array}{r}1 \\
28 \\
29\end{array}$ & $\begin{array}{r}970.770 \\
99.472\end{array}$ & 9.759 & $.004 a$ \\
\hline 2 & $\begin{array}{l}\text { Regression } \\
\text { Residual } \\
\text { Total }\end{array}$ & $\begin{array}{l}1471.443 \\
2284.557 \\
3756.000\end{array}$ & $\begin{array}{r}2 \\
27 \\
29\end{array}$ & $\begin{array}{r}735.721 \\
84.613\end{array}$ & 8.695 & $.001^{b}$ \\
\hline 3 & $\begin{array}{l}\text { Regression } \\
\text { Residual } \\
\text { Total }\end{array}$ & $\begin{array}{l}2440.912 \\
1315.088 \\
3756.000\end{array}$ & $\begin{array}{r}3 \\
26 \\
29\end{array}$ & $\begin{array}{r}813.637 \\
50.580\end{array}$ & 16.086 & $.000^{\circ}$ \\
\hline
\end{tabular}

a. Predictors: (Constant), BRANX

b. Predictors: (Constant), BRANX, BENEX2

c. Predictors: (Constant), BRANX, BENEX2, COMPXI

d. Dependent Variable: ENGAY

Coefficients $a$

\begin{tabular}{|c|c|c|c|c|c|c|}
\hline \multirow{2}{*}{\multicolumn{2}{|c|}{ Model }} & \multicolumn{2}{|c|}{$\begin{array}{l}\text { Unstandardized } \\
\text { Coefficients }\end{array}$} & \multirow{2}{*}{\begin{tabular}{|c|}
$\begin{array}{c}\text { Standardized } \\
\text { Coefficients }\end{array}$ \\
Beta
\end{tabular}} & \multirow[b]{2}{*}{$\dagger$} & \multirow[b]{2}{*}{ Sig. } \\
\hline & & B & Std. Error & & & \\
\hline \multirow[t]{2}{*}{1} & (Constant) & 34.269 & 15.705 & & 2.182 & .038 \\
\hline & BRANX & .549 & .176 & .508 & 3.124 & .004 \\
\hline \multirow[t]{3}{*}{2} & (Constant) & 22.475 & 15.274 & & 1.471 & .153 \\
\hline & BRANX & .499 & .164 & .462 & 3.051 & .005 \\
\hline & BENEX2 & .160 & .066 & .368 & 2.433 & .022 \\
\hline \multirow[t]{4}{*}{3} & (Constant) & 1.874 & 12.713 & & .147 & .884 \\
\hline & BRANX & .794 & .143 & .735 & 5.543 & .000 \\
\hline & BENEX2 & .347 & .066 & .799 & 5.226 & .000 \\
\hline & COMPX 1 & -.710 & .162 & -.740 & -4.378 & .000 \\
\hline
\end{tabular}


Coefficients $a$

\begin{tabular}{|c|c|c|c|c|c|c|}
\hline \multirow{2}{*}{\multicolumn{2}{|c|}{ Model }} & \multicolumn{2}{|c|}{$\begin{array}{l}\text { Unstandardized } \\
\text { Coefficients }\end{array}$} & \multirow{2}{*}{\begin{tabular}{|c|}
$\begin{array}{c}\text { Standardized } \\
\text { Coefficients }\end{array}$ \\
Beta
\end{tabular}} & \multirow[b]{2}{*}{$\dagger$} & \multirow[b]{2}{*}{ Sig. } \\
\hline & & B & Std. Error & & & \\
\hline \multirow[t]{2}{*}{1} & (Constant) & 34.269 & 15.705 & & 2.182 & .038 \\
\hline & BRANX & .549 & .176 & .508 & 3.124 & .004 \\
\hline \multirow[t]{3}{*}{2} & (Constant) & 22.475 & 15.274 & & 1.471 & .153 \\
\hline & BRANX & .499 & .164 & .462 & 3.051 & .005 \\
\hline & BENEX2 & .160 & .066 & .368 & 2.433 & .022 \\
\hline \multirow[t]{4}{*}{3} & (Constant) & 1.874 & 12.713 & & .147 & .884 \\
\hline & BRANX & .794 & .143 & .735 & 5.543 & .000 \\
\hline & BENEX2 & .347 & .066 & .799 & 5.226 & .000 \\
\hline & COMPX 1 & -.710 & .162 & -.740 & -4.378 & .000 \\
\hline
\end{tabular}

a. Dependent Variable: ENGAY

Excluded Variablesc

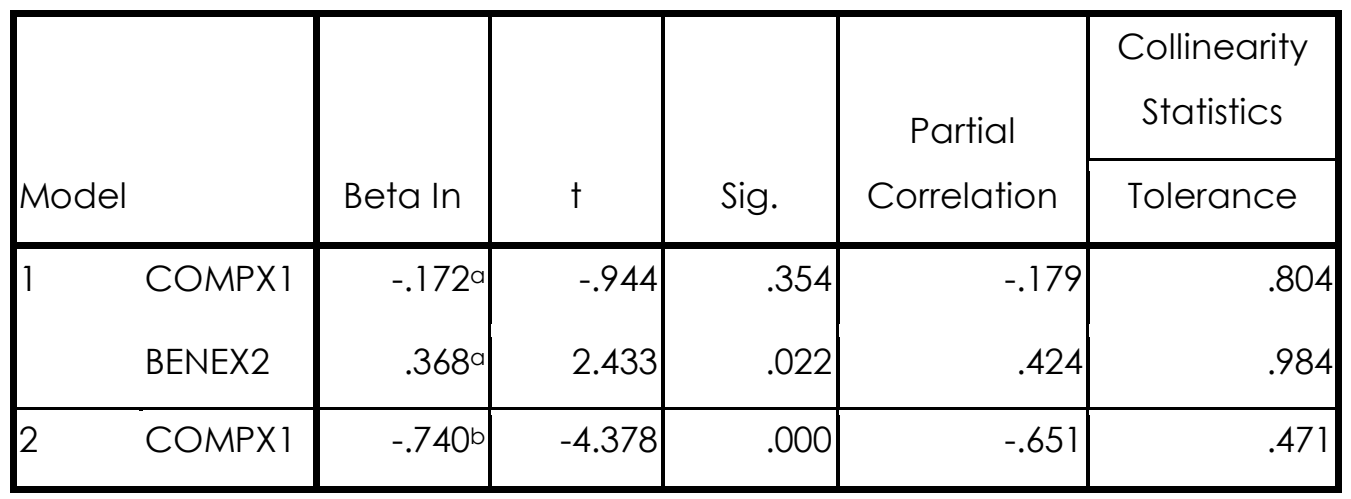

a. Predictors in the Model: (Constant), BRANX

b. Predictors in the Model: (Constant), BRANX, BENEX2

c. Dependent Variable: ENGAY

In another research question we also wanted to know how compensation and benefit having a relationship with any other variables that are organisation brand and employee engagement. We are as well using linier regression method to see the effect compensation 
and benefit have to other two variables. Determinant coefficient of compensation to employee engagement is 0.008 which means that $8 \%$ of compensation defined employee engagement while the other percentation defined by another variables. Siginificance coefficient is 0.646 and that means compensation have no effect on employee engagement. Different findings in benefit which determinant coefficient is 0.182 . that means $18,2 \%$ employee engagement is defned by benefit with significance level on 0.019 that is less than 0.05 . So the benefit variable have some effect on employee engagement.

Table 6. SPSS Result of Linier Regression Compensation to Employee Engagement Model Summary

\begin{tabular}{|l|r|r|r|r|}
\hline Model & $R$ & R Square & \multicolumn{1}{c|}{$\begin{array}{c}\text { Adjusted R } \\
\text { Square }\end{array}$} & $\begin{array}{c}\text { Std. Error of } \\
\text { the Estimate }\end{array}$ \\
\hline 1 & $.087 \mathrm{a}$ & .008 & -.028 & 11.538 \\
\hline
\end{tabular}

a. Predictors: (Constant), COMPX1

ANOVAb

\begin{tabular}{|c|c|c|c|c|c|}
\hline Model & $\begin{array}{l}\text { Sum of } \\
\text { Squares }\end{array}$ & $\mathrm{df}$ & Mean Square & $F$ & Sig. \\
\hline $\begin{array}{l}\text { Regression } \\
\text { Residual } \\
\text { Total }\end{array}$ & $\begin{array}{r}28.666 \\
3727.334 \\
3756.000\end{array}$ & $\begin{array}{r}1 \\
28 \\
29\end{array}$ & $\begin{array}{r}28.666 \\
133.119\end{array}$ & .215 & $.646^{\circ}$ \\
\hline
\end{tabular}

a. Predictors: (Constant), COMPXI

b. Dependent Variable: ENGAY 
Coefficients $a$

\begin{tabular}{|rr|r|r|r|r|r|}
\hline \multirow{2}{*}{ Model } & \multicolumn{2}{|c|}{$\begin{array}{c}\text { Unstandardized } \\
\text { Coefficients }\end{array}$} & $\begin{array}{c}\text { Standardized } \\
\text { Coefficients }\end{array}$ & & \\
\cline { 2 - 5 } & \multicolumn{1}{|c|}{ B } & Std. Error & \multicolumn{1}{c|}{ Beta } & \multicolumn{1}{c|}{+} & \multicolumn{1}{c|}{ Sig. } \\
\hline 1 & (Constant) & 80.092 & 6.612 & & 12.113 & .000 \\
COMPX1 & .084 & .181 & .087 & .464 & .646 \\
\hline
\end{tabular}

a. Dependent Variable: ENGAY

Table 7. SPSS Result of Linier Regression Benefit to Employee Engagement Model Summary

\begin{tabular}{|l|r|r|r|r|}
\hline Model & $R$ & R Square & \multicolumn{1}{c|}{$\begin{array}{c}\text { Adjusted R } \\
\text { Square }\end{array}$} & $\begin{array}{c}\text { Std. Error of } \\
\text { the Estimate }\end{array}$ \\
\hline 1 & .4279 & .182 & .153 & 10.475 \\
\hline
\end{tabular}

a. Predictors: (Constant), BENEX2

ANOVAb

\begin{tabular}{|c|c|c|c|c|c|c|}
\hline \multicolumn{2}{|c|}{ Model } & $\begin{array}{l}\text { Sum of } \\
\text { Squares }\end{array}$ & $\mathrm{df}$ & Mean Square & $\mathrm{F}$ & Sig. \\
\hline \multirow[t]{3}{*}{1} & Regression & 683.653 & 1 & 683.653 & 6.231 & $.019 a$ \\
\hline & Residual & 3072.347 & 28 & 109.727 & & \\
\hline & Total & 3756.000 & 29 & & & \\
\hline
\end{tabular}

a. Predictors: (Constant), BENEX2

b. Dependent Variable: ENGAY

Coefficients $a$

\begin{tabular}{|c|c|c|c|c|c|}
\hline \multirow[b]{2}{*}{ Model } & \multicolumn{2}{|c|}{$\begin{array}{l}\text { Unstandardized } \\
\text { Coefficients }\end{array}$} & \multirow{2}{*}{\begin{tabular}{|c|}
$\begin{array}{c}\text { Standardized } \\
\text { Coefficients }\end{array}$ \\
Beta
\end{tabular}} & \multirow[b]{2}{*}{$\dagger$} & \multirow[b]{2}{*}{ Sig. } \\
\hline & B & Std. Error & & & \\
\hline (Constant) & 64.143 & 7.793 & & 8.231 & .000 \\
\hline BENEX2 & .185 & .074 & .427 & 2.496 & .019 \\
\hline
\end{tabular}




\section{Coefficientsa}

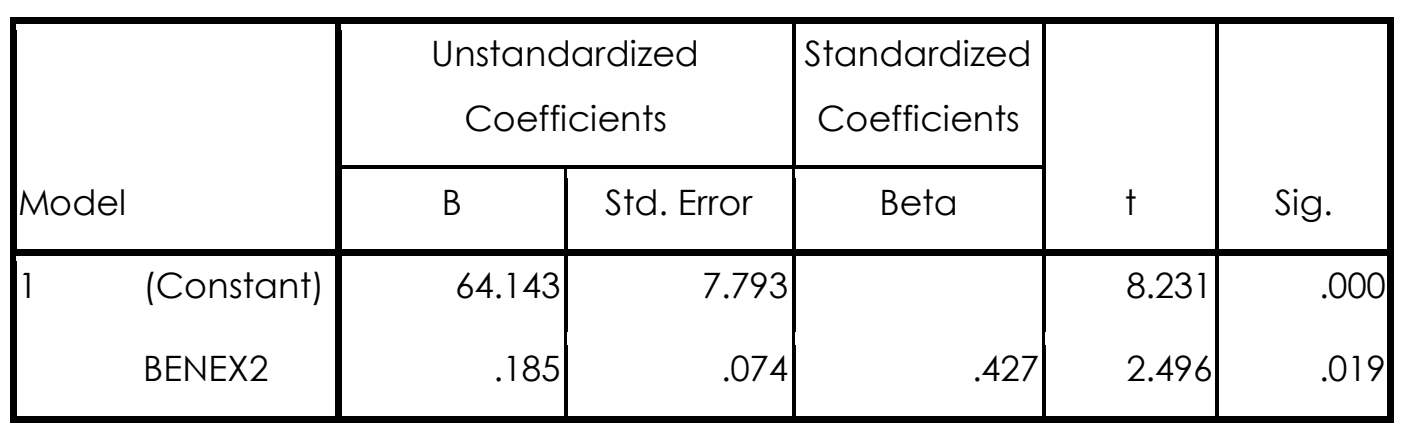

a. Dependent Variable: ENGAY

About benefit effecting in organisation brand, the determinant coefficient resulting in -0.19 with significance level on 0.504. it means that benefit have no effect on organisation brand. In the other side, compensation that in relation with employee engagement is having no effect, in organisation brand compensation show some significance level on 0.14 , less than 0.05 with determinant coefficient in 0.168 or $16,8 \%$.

Table 8. SPSS Result of Linier Regression Compensation to Organisation Brand Model Summary

\begin{tabular}{|l|r|r|r|r|}
\hline Model & $R$ & $R$ Square & \multicolumn{1}{c|}{$\begin{array}{c}\text { Adjusted R } \\
\text { Square }\end{array}$} & $\begin{array}{l}\text { Std. Error of } \\
\text { the Estimate }\end{array}$ \\
\hline 1 & .4430 & .196 & .168 & 9.608 \\
\hline
\end{tabular}

a. Predictors: (Constant), COMPXI

ANOVAb

\begin{tabular}{|c|c|c|c|c|c|}
\hline Model & $\begin{array}{l}\text { Sum of } \\
\text { Squares }\end{array}$ & $d f$ & Mean Square & $\mathrm{F}$ & Sig. \\
\hline $\begin{array}{l}\text { Regression } \\
\text { Residual } \\
\text { Total }\end{array}$ & $\begin{array}{r}631.568 \\
2584.732 \\
3216.300\end{array}$ & $\begin{array}{r}1 \\
28 \\
29\end{array}$ & $\begin{array}{r}631.568 \\
92.312\end{array}$ & 6.842 & $.014 a$ \\
\hline
\end{tabular}

a. Predictors: (Constant), COMPXI

b. Dependent Variable: BRANX 
Coefficients $a$

\begin{tabular}{|rr|r|r|r|r|r|}
\hline \multirow{2}{*}{ Model } & \multicolumn{2}{|c|}{$\begin{array}{c}\text { Unstandardized } \\
\text { Coefficients }\end{array}$} & $\begin{array}{c}\text { Standardized } \\
\text { Coefficients }\end{array}$ & & \\
\cline { 2 - 5 } & \multicolumn{1}{|c|}{ B } & Std. Error & \multicolumn{1}{c|}{ Beta } & \multicolumn{1}{c|}{+} & \multicolumn{1}{c|}{ Sig. } \\
\hline 1 & (Constant) & 75.048 & 5.506 & & 13.630 & .000 \\
& COMPX1 & .393 & .150 & .443 & 2.616 & .014 \\
\hline
\end{tabular}

a. Dependent Variable: BRANX

Table 9. SPSS Result of Linier Regression Benefit to Organisation Brand Model Summary

\begin{tabular}{|l|r|r|r|r|}
\hline Model & \multicolumn{1}{|c|}{$R$} & R Square & \multicolumn{1}{c|}{$\begin{array}{c}\text { Adjusted R } \\
\text { Square }\end{array}$} & $\begin{array}{c}\text { Std. Error of } \\
\text { the Estimate }\end{array}$ \\
\hline 1 & $.127 \mathrm{a}$ & .016 & -.019 & 10.631 \\
\hline
\end{tabular}

a. Predictors: (Constant), BENEX2

ANOVAb

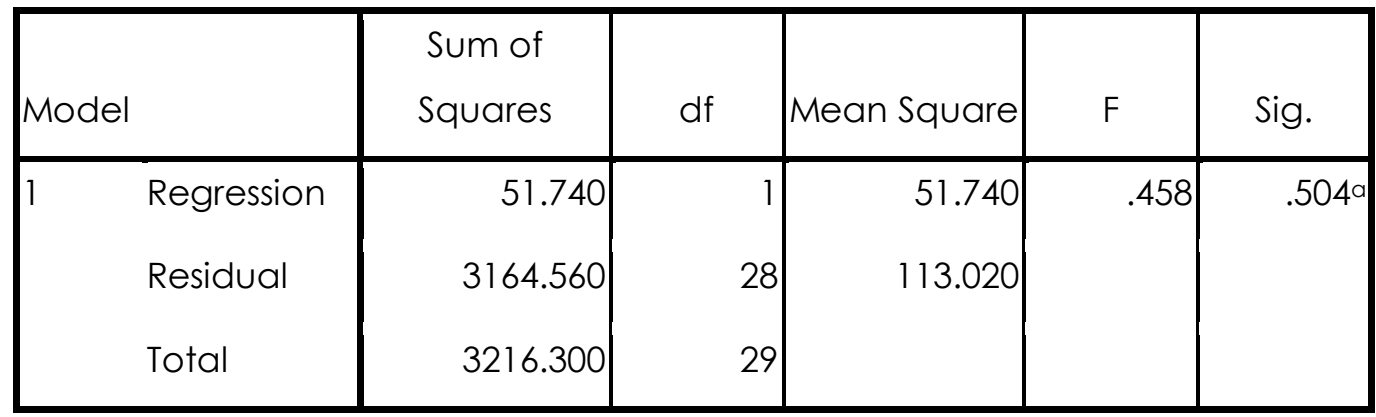

a. Predictors: (Constant), BENEX2

b. Dependent Variable: BRANX

Coefficients ${ }^{a}$

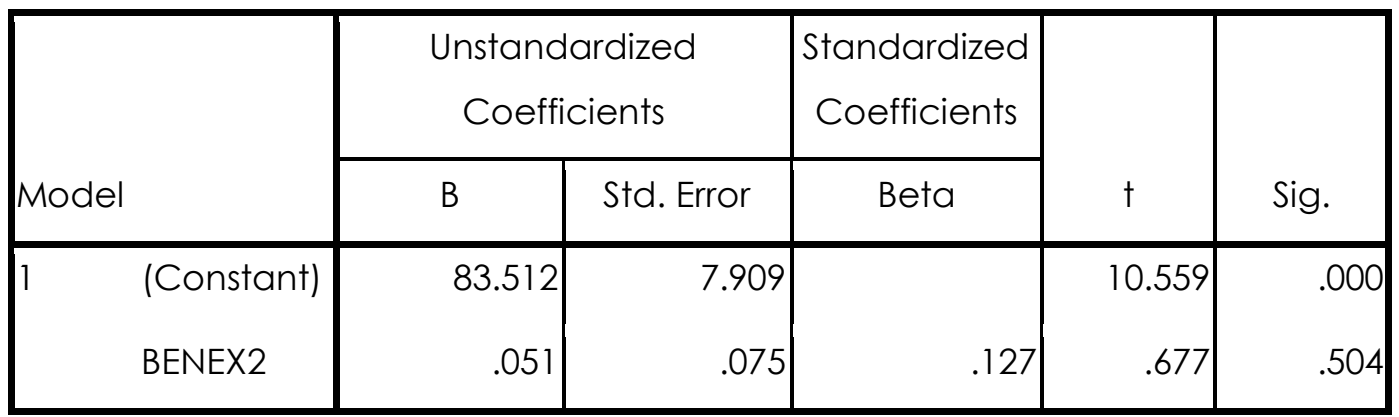


Coefficientsa

\begin{tabular}{|c|c|c|c|c|c|}
\hline \multirow[b]{2}{*}{ Model } & \multicolumn{2}{|c|}{$\begin{array}{c}\text { Unstandardized } \\
\text { Coefficients }\end{array}$} & \multirow{2}{*}{\begin{tabular}{|c|}
$\begin{array}{c}\text { Standardized } \\
\text { Coefficients }\end{array}$ \\
Beta
\end{tabular}} & \multirow[b]{2}{*}{$\dagger$} & \multirow[b]{2}{*}{ Sig. } \\
\hline & B & Std. Error & & & \\
\hline (Constant) & 83.512 & 7.909 & & 10.559 & .000 \\
\hline BENEX2 & .051 & .075 & .127 & .677 & .504 \\
\hline
\end{tabular}

a. Dependent Variable: BRANX

\section{SUMMARY AND CONCLUSIONS}

From this research we can conclude that organisation brand is indeed have some effect on employee engagement, whether it stands alone or with another mediating variable. In this research, mediating variable we use result in only on that have a real mediating role between organisation brand and employee engagement. That is benefit. But when we are seeing compensation and benefit as an independent variable to organisation brand or to employee engagement, compensation have no effect on employee engagement but benefit have, compensation have effect on organisation brand but benefit doesn't have. In summary, hypotheses we proposed earlier is reaching to this result.

Table 10. Hypotheses Result

\begin{tabular}{|l|l|}
\hline \multicolumn{1}{|c|}{ Hypotheses } & \multicolumn{1}{|c|}{ Result } \\
\hline $\mathrm{H} 1:$ Organization brand have effect on employee engagement. & Accepted \\
\hline $\begin{array}{l}\mathrm{H} 2 \mathrm{a}: \text { Compensation is a mediating variable in the effect of organization } \\
\text { brand to employee engagement }\end{array}$ & Rejected \\
\hline $\begin{array}{l}\mathrm{H} 2 \mathrm{~b}: \text { Benefit is a mediating variable in the effect of organization brand to } \\
\text { employee engagement }\end{array}$ & Accepted \\
\hline $\mathrm{H} 3 \mathrm{a}:$ Compensation is the factor affecting employee engagement & Rejected \\
\hline $\mathrm{H} 3 \mathrm{~b}:$ Benefit is the factor affecting employee engagement & Accepted \\
\hline $\mathrm{H} 3 \mathrm{C}:$ Compensation is the factor affecting organization brand & Accepted \\
\hline $\mathrm{H} 3 \mathrm{~d}:$ Benefit is the factor affecting organization brand & Rejected \\
\hline
\end{tabular}

We can conclude that organization brand have effect on employee engagement because when employee have internalized the brand on their self, they will have some attachment to the company. It will result in engagement between the employee and company. While compensation are failed to be mediating variable but benefit are successful, it might be 


\section{Astri Utami Indriyani \\ Ignatius Heruwasto}

because employee in start-up business are triggered by additional reward they get to support their daily needs, related to themselves or to family.

Further research needed to see the relationship between those variables. Because it haven't exist such research about start-up business especially in context of compensation and benefit. Different resources and participant will add more knowledge in academic world.

\section{REFERENCES}

Abel, M 2013. 'The social and financial benefits of developing employee satisfaction'. International Journal of Management \& Information Systems, Vol. 17, No. 2, pp. 83-88.

Abratt, R and Kleyn, N 2012. 'Corporate identity, corporate branding and corporate reputations', European Journal of Marketing, Vol. 46, No 7-8, pp. 1048-1063.

Ahmad, N., labal, N., Kanwal R., Javed. H, and Javed. K 2014. 'The mediating role of employee engagement in relationship of internal branding and brand experience: Case of service organizations of Dera Ghazi Khan'. International Journal of Information Business and Management, Vol. 6, No 4, pp. 26-41.

Anitha, J 2014. 'Determinants of employee engagement and their impact on employee performance'. International Journal of Productivity and Performance Management, Vol. 63, N. 3, pp. 308-323

Blumenthal, D 2011 . Internal branding: does it improve employees' quality of life? Institute for Brand Leadership.

Boyd, $G$ and Sutherland, M 2006. 'Obtaining employee commitment to living the brand of the organization. Journal of Business Management, Vol. 37, No 1, pp. 209-216

Condos, JR 2016. The relationshop between transformational leadership and employee engagement in Southwest Lovisiana Law Firms. Dissertation, Northcentral University, Northcentral.

Council, C. L. (2004, January 1). Driving Performance and Retention Through Employee Engagement. Retrieved from Corporate Leadership: www.corporateleadershipcouncil.com

Hewitt, A. (2015, June 1). Say, Stay, or Strive? Unleash the Engagement Outcome You Need. Retrieved from AON Hewitt: http://aonhewitt.com

Hewitt, A. (2016, 12 1). 2016 Trends in Global Employee Engagement. Retrieved from AON Hewitt: http://www.aonhewitt.com 
Mahatma, R. (2013, June 19). Apakah Definisi Startup Itu ? Kenapa Sering Dikaitkan Dengan Teknologi Atau Internet. Retrieved from StartupBisnis: http://startupbisnis.com/apakahdefinisi-startup-itu-kenapa-sering-dikaitkan-dengan-teknologi-atau-internet/

Martocchio, J. J. 2013. Strategic Compensation: A Human Resource Management Approach ( $7^{\text {th }}$ ed). New Jersey: Pearson.

Milkovich, G., Newman, J., Gerhart, B. 2011 . Compensation (1 $1^{\text {th }}$ ed). New York: McGraw-Hill Irwin.

Murphy, K. J.: 1999, 'Executive Compensation', in O. Ashenfelter and D. Card (eds.), Handbook of Labour Economics (Elsevier, Amsterdam), pp. 2485-2563.

Pratama, A. H. (2016, December 14). Kilas Balik Perkembangan E-Commerce di Indonesia Sepanjang Tahun 2016. Retrieved from Tech in Asia: https://id.techinasia.com/kilasbalik-perkembangan-e-commerce-di-tahun-2016

Punjaisri, K., Wilson. A., and Evanschitzky. H 2009. 'Internal branding to influence employees' brand promise delivery: a case study in Thailand' Journal of Service Management, Vol. 20, No 5, pp. 561-579.

Punjaisri, K., Evanschitzky. H, and Wilson. A 2009. 'Internal branding: an enabler of employees' brand-supporting behaviours'. Journal of Service Management, Vol. 20, No. 2, pp. 209-226. 